\title{
THANKS TO ReVIEWERS
}

The Editors gratefully acknowledge the generous assistance of the following individuals (apart from the Editorial Board) who reviewed manuscripts in 2000.

Cristian Achim
Masanori Aikawa
Richard Ajioka
Kari Alitalo
Gianfranco Alpini
Henry Appelman
Douglas Arenberg
Phillip Askenase
Craig Atwood
Hellmut G Augustin
Ellis D Avner
Thomas M Badger
Marco Baggiolini
William Baldwin
Steven P Balk
Lawrence S Baskin
Marc D Basson
Robert Baughman
Bruce Baum
Victoria Bautch
M Flint Beal
Joseph S Beckman
Richard H Bell
Laura Benjamin
Merrill Benson
Richard Benya
Gideon Berke
Merton R Bernfield
David E Birk
Elizabeth H Blackburn
Francesco Blasi
C Richard Boland
Wayne A Border
Silvano Bosari
David G Bostwick

Neil A Bradbury

John Bradley

Janet L Brandsma

Jonathan Braun

Jan L Breslow

Thomas Brock

Inka Brockhausen

Leena Bruckner-Tuderman

Clayton A Buck

Gerard Burrow

Paul Cairns

Julie Campbell

Michael Carrithers

Darryl Carter

Richard Cartun

Sally E Carty

Martha Cathcart

Edgar S Cathcart

Marco Cattaneo

Michael Centrella

Israel F Charo

Yi-Wen Chen

Kathleen R Cho

Michael J Clare-Salzler

Lauren Cohn

V Peter Collins

Galen Cortina

Marta E Couce

Joseph Craft

James D Crapo

lan N Crispe

Gerald R Cunha

Mohamed Daha

Thomas Dengler

Alexis Desmouliere
Daniel J Donoghue

Colin Duckett

Stephen C Edberg

Dylan Edwards

Marie Egan

Tom Ehling

Charis Eng

Peter B Ernst

Angels Fabra

Christiane Ferran

Erol Fikrig

Susan J Fisher

J Kevin Foskett

Harris Foster

Velia Fowler

Robert I Fox

Werner W Franke

Wilbur A Franklin

Michael R Freeman

Rafi Fridman

Glauco Frizzera

Dai Fukumura

Michael Gale

Warren Gallin

Edward P Gelmann

Gregory G Germino

Mary E Gerritsen

Reza Ghohestani

Ronald Ghossein

Cecilia Giachelli

Steven Goodison

D Neil Granger

Georges Grau

Patricia Greenwel

Roberto Groszmann 
Anna Gukovskaya

Emanuela Gussoni

Thomas A Haas

Zishan A Haroon

Nancy Lee Harris

Christian C Haudenschild

Peter Herrlich

Denise C Hocking

Eric Hoffman

Shawn E Holt

Robert Homer

Mark Horowitz

Gregg Howe

Linda Hsi

Pei Hui

Bonnie Hylander

David Ingbar

Giorgio Inghirami

David F Jarrard

Jeremy $R$ Jass

David Johnson

Barry Kacinski

Marshall Kadin

Olli Kallioniemi

Osami Kanagawa

Stephen Karlik

Suresh Karne

Michael Kashgarian

David Kaufman

Karen Kaul

Paula B Kavathas

Tammy Kielian

George King

Bonnie King

Hynda K Kleinman

Alisa E Koch

Olivier Kocher

Elise C Kohn

Ron Rieger Kopito

Gyula Kovacs

Paul H Krebsbach

Jeffrey Kreisberg
Wilhelm Kriz

Richard Kroczek

Charles Kuhn

Thomas S Kupper

JP Kushner

Claude Labrie

Marc Ladanyi

Michael MC Lai

Marie Landry

B Lowell Langille

Lucia Languino

Pierre Laurent-Puig

Jack Lawler

Yuri Lazebnik

Edward Le Cluyse

Charles Lee

Leslie Leinwand

Peter Libby

Michael $\mathrm{P}$ Lisanti

Massimo Loda

Elizabeth Luna

Stewart D Lyman

JC Machada

Peter Maisonpierre

Mark Majesky

Guido Majno

A Mansukhani

M Louise Markert

Pierre Massion

Louis Matis

Lynn M Matrisian

Mark Mattie

Robert Matusik

Jon McCullers

Hugh O McDevitt

Sandra McLeskey

Myron R Melamed

Didier Merlin

Paolo Mignatti

Christopher Miller

John Minna

Elizabeth Montgomery
Ethel Moustacchi

HK Muller-Hermelink

John Murren

Mohamad Musavi

Markus Müschen

S Muttukrishna

Linda Kay Myers

William O Myers

Frederick Naftolin

Karl Nath

Lauren Nathan

Volker Nickeleit

Soren Nielsen

Yuri Nikiforov

Matthew Nugent

Timothy J O'Leary

Janet M Oliver

Mitchell A Olman

Terry J Opgenorth

Timothy Oury

David L Page

George E Palade

Charles A Parkos

Thomas Parks

Paul Pattengale

Michael S Pepper

Archibald Perkins

Manuel Perucho

Peter Petzelbauer

Sem Hin Phan

Halit Pinar

Bruce R Pitt

Daniel K Podolsky

Alan Poritz

Thomas Pretlow

John Prins

Leena Pulkkinen

Mazin B Qumsiyeh

Leticia Qunitanilla-Martin

Hari A Reddi

Michael Reiss

Xiang-Dong Ren 
Miguel Reyes-Mugica

Daniel B Rifkin

David J Riley

Piero Rinaldo

Enrique R Rodriguez-Boulan

Elliot Rosen

Seymour Rosen

Thomas Ruenger

Raymond R Russell

Sami I Said

Elaine Schattner

Jeffrey Schechner

William Schlaepfer

Ann Marie Schmidt

William Schnaper

Barbara G Schneider

Stuart Schnitt

Uwe Schoenbeck

Daniel D Sedmak

Steven S Segal

Ephraim Sehayek

Donald R Senger

Manuel Serrano

Raquel Seruca

William Sessa

Barbara Sherry

Robert Sherwin

le-Ming Shih
Mark Shlomchik

Bjorn Skytting

Brian R Smith

Solomon Snyder

Ivan Stamenkovic

Michael Steer

Maryalice Stetler-Stevenson

Stephen StohIman

Phillip Stone

John P Sundberg

Sandor Szabo

Gerald Taborsky

Chris Takimoto

Giovanni Tallini

Ronald P Taylor

George Tellides

Martin Tenniswood

Brent A Tetri

Jeffrey Trent

Jenifer Turco

Jouni Uitto

Victor Van Hinsbergh

George F Vande Woude

John $P$ Veinot

Arthur Veis

Mark A Velleca

Agnes Vignery

Stanley A Vinores
Kenneth Walsh

Heinrich Walt

JS Wang

Aaron Waxman

H Christian Weber

Robert Webster

I Bernard Weinstein

Robert M Weiss

Sharon W Weiss

Babette Weksler

Li Wen

Rudi GJ Westendorp

Cornelia Weyond

Marcia Wheeler

James G White

Carl White

Josiah Wilcox

Stuart K Williams

Susan Wong

George Yancopoulos

Gang-Qing Yao

Andreas Zeiher

Daniel Zelterman

Pumin Zhang

David Zhao

Marina Ziche

Stanley Zucker 